\title{
Tertiary osteochondral defect of the talus treated by a novel contoured metal implant
}

\author{
Christiaan J. A. van Bergen • Mikel L. Reilingh • \\ C. Niek van Dijk
}

Received: 11 November 2010/Accepted: 21 February 2011/Published online: 16 March 2011

(C) The Author(s) 2011. This article is published with open access at Springerlink.com

\begin{abstract}
The primary treatment of most osteochondral defects of the talus is arthroscopic debridement and bone marrow stimulation. There is no optimal treatment for large lesions or for those in which primary treatment has failed. We report a 20-year-old female patient with persistent symptoms after two previous arthroscopic procedures. Computed tomography showed a cystic defect of the medial talar dome, sized $17 \times 8 \times 8 \mathrm{~mm}$. The patient was treated with a novel contoured metal implant. At 1 and 2 years after surgery, the patient reported considerable reduction in pain and had resumed playing korfball at competitive level.

Level of evidence IV.
\end{abstract}

Keywords Osteochondral lesions - Bone cyst - Talus . Ankle $\cdot$ Metal implant $\cdot$ Treatment

\section{Introduction}

Arthroscopic debridement and bone marrow stimulation is considered the primary treatment of osteochondral talar defect (OCD) and yields $85 \%$ success [26]. In case of failure of the primary treatment, current secondary treatment options include repeat arthroscopic debridement and bone marrow stimulation, osteochondral autograft transfer, autogenous cancellous bone graft, and autologous chondrocyte implantation [4, 8, 9, 20, 23]. However, these techniques are sometimes associated with donor-site morbidity or involve two-stage surgery [2, 3, 13, 14].

C. J. A. van Bergen - M. L. Reilingh ( $₫)$. C. N. van Dijk

Academic Medical Center, University of Amsterdam,

Amsterdam, The Netherlands

e-mail: M.L.Reilingh@amc.nl
In order to treat OCDs of the medial talar dome after failed primary treatment, a contoured articular inlay implant (HemiCAP ${ }^{\circledR}$, Arthrosurface Inc., Franklin, MA, USA) with a diameter of $15 \mathrm{~mm}$ was developed. Fifteen articular component offset sizes are available, based on the surface anatomy of the medial talar dome. In a cadaveric study, the offset sizes were found appropriate for a variety of talar specimens. Furthermore, it was shown that a slightly recessed implantation level prevents high contact pressures [22]. Clinical goals of the implant are to offer relief of pain and swelling, return to activity, and prevent (further) cyst formation.

A two-year result of the first patient who was treated with the novel implant is reported.

\section{Case report}

A 20-year-old female was referred with an OCD of the right talus. Her symptoms had started after a supination trauma of the ankle. She had been treated twice in the referring hospital. During both procedures, the defect was excised and curetted through anterior arthroscopic surgery. The last procedure was 1 year and 2 months before her presentation.

At presentation, the patient's predominant symptom was pain deep in the ankle joint during and after activity. She used to play korfball at competitive level but had stopped due to the pain. Korfball is a mixed gender team ball game, similar to mixed netball and basketball, and is very popular in the Netherlands.

At physical examination, leg alignment was neutral, the range of motion was normal and symmetric, and both ankle joints were stable. There was no recognizable tenderness on palpation. 
Fig. 1 Preoperative anteroposterior mortise view and lateral radiographs of the affected ankle showing a radiolucent osteochondral defect in the medial talar dome (arrow)

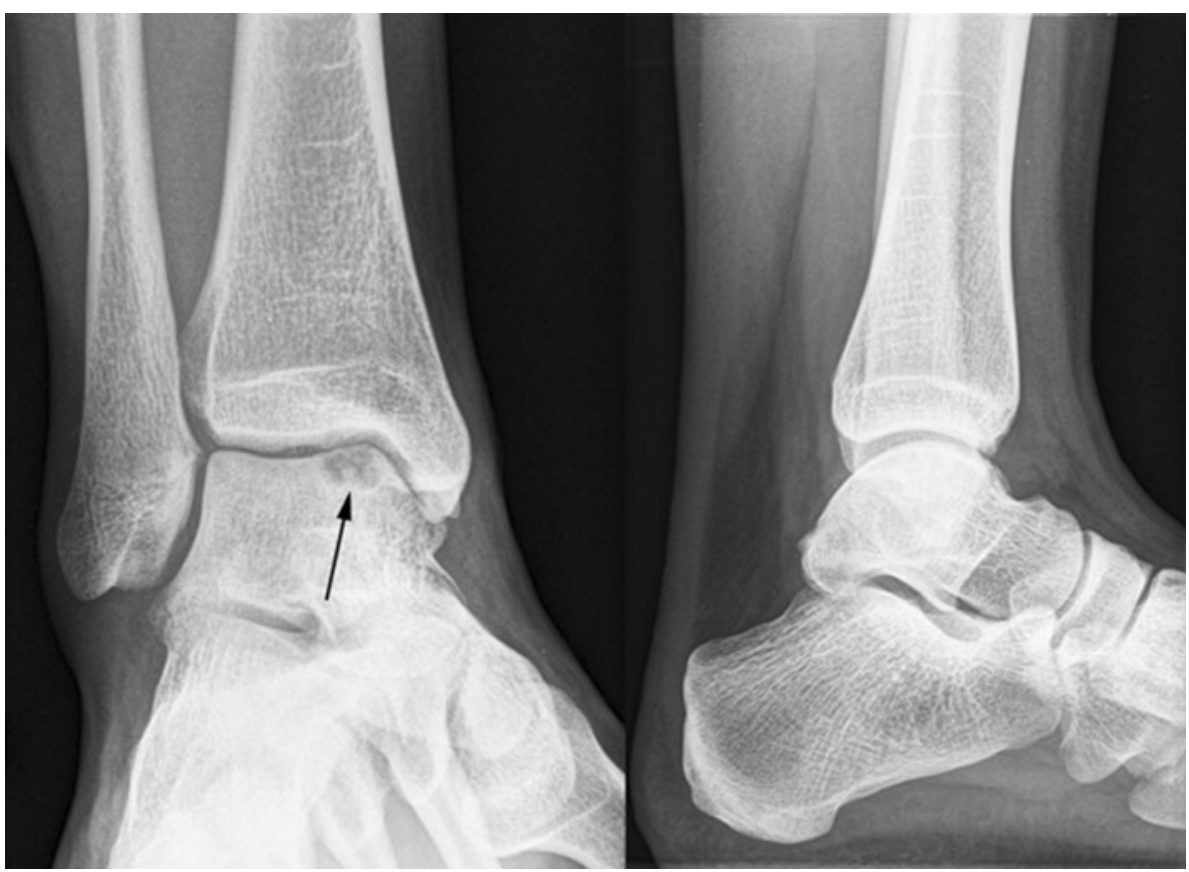

Radiographs of the right ankle revealed a large cystic osteochondral defect of the medial talar dome (Fig. 1). A computed tomography scan confirmed the osteochondral defect, measuring $17 \times 8 \times 8 \mathrm{~mm}$, accompanied by some small subchondral cysts and a loose fragment. After informed consent was obtained, the patient was scheduled for surgery.

\section{Operative technique}

A curved skin incision was made over the medial malleolus. After two screw holes were predrilled in the medial malleolus, a medial malleolar osteotomy was made at an angle of 30 degrees relative to the long tibial axis [21], exposing the osteochondral defect (Fig. 2a).

The defect was debrided. Utilizing a drill guide, a guide pin was placed into the center of the defect, perpendicular to the curvature of the medial talar dome. The cannulated screw was inserted. A contact probe was used to determine the radius of curvature; an offset size of $0.5 \times 3.5 \mathrm{~mm}$ was measured. A matching reamer prepared the site for placement of the articular component. A trial articular component allowed for final verification of proper fit (Fig. 2b). The selected articular component was placed on the screw (Fig. 2c). It was impacted, thereby engaging the taper interlock (Fig. 2d). The osteotomy was fixated with two 3.5-mm lag screws, and the wound was closed.

The postoperative management was initiated with a plaster cast for 1 week and continued with a functional brace (Walker, Össur, Son en Breugel, the Netherlands) for
5 weeks. After this 6-week period, radiographs of the operated ankle confirmed consolidation of the malleolar osteotomy. Physical therapy was prescribed to advance to full weight bearing in 1 month.

\section{Outcome}

The patient recovered well from surgery. Initially, there was a small area of numbness about the scar but this had resolved at 3 months follow-up. The patient was able to play korfball at the preinjury level after 1 year and continued to play at this level at 2 years follow-up. The sagittal range of motion arc remained slightly reduced: $15^{\circ}$ of dorsiflexion to $45^{\circ}$ of plantar flexion compared to $20^{\circ}$ of dorsiflexion to $45^{\circ}$ of plantar flexion in the left ankle.

Using numeric rating scales, the patient preoperatively rated the pain as 0 when at rest, 8 when walking, and 10 when running. These numbers improved to 0,0 , and 1 at final follow-up, respectively. The Foot and Ankle Outcome Score [15] improved from preoperatively to final follow-up on four of five subscales; the subscale "other symptoms" decreased due to subjective inability to fully straighten and bend the ankle. The American Orthopaedic Foot and Ankle Society ankle-hindfoot score [11] improved from 74 preoperatively to 90 at 1 and 2 years.

Radiographs were obtained at all follow-up visits. The implant remained in position, and there were no signs of osteolysis or progressive degenerative changes in the ankle joint (Fig. 3). 

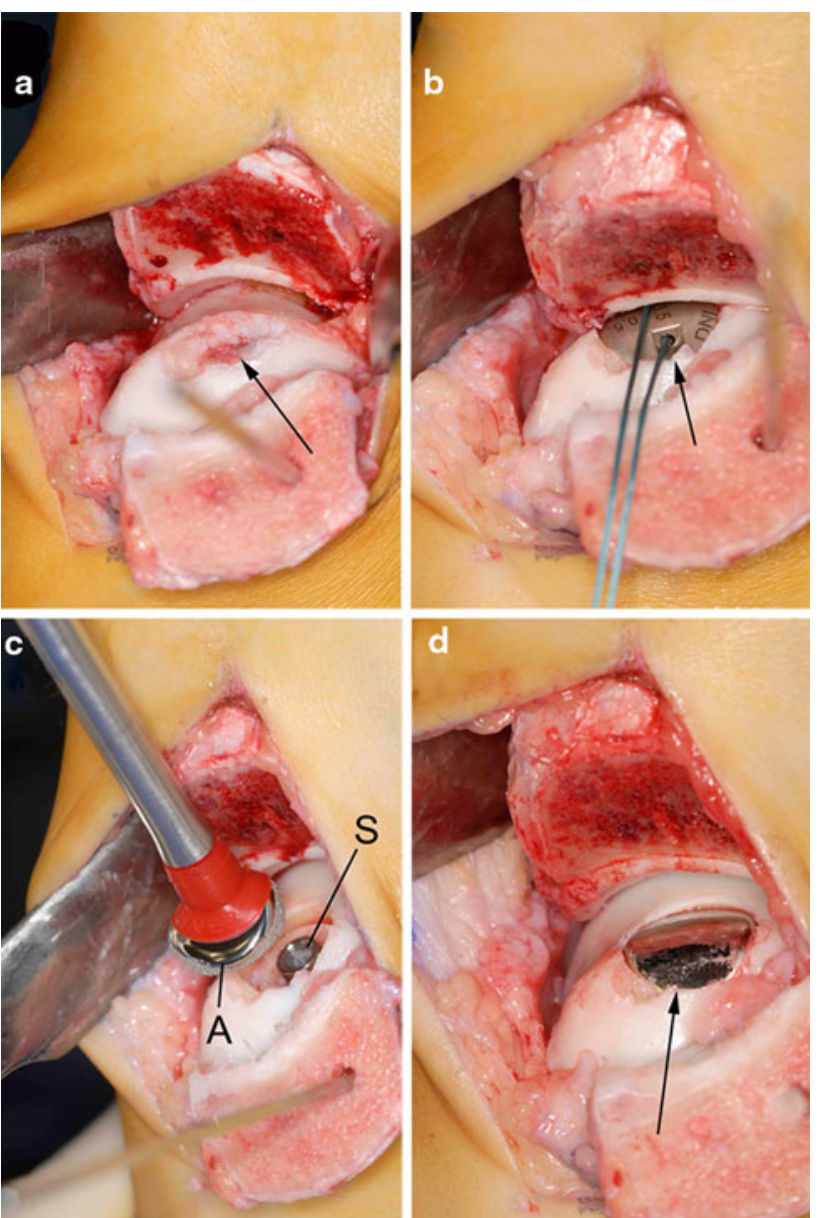

Fig. 2 Intraoperative pictures of the operative technique. a The talar lesion was exposed through an oblique medial malleolar osteotomy, and the necrotic fragment was excised (arrow). b After the insertion of a screw and the determination of the appropriate offset sizes, a trial articular component (arrow) was placed. c The final articular component (A) was orientated in the correct plane and placed on the screw (S). d Final view of the talus after engagement of the articular component (arrow). Note that the edges of the implant are slightly recessed compared to the adjacent cartilage level

\section{Discussion}

This prospective case report is the first clinical report of a metal implant for OCDs of the talus and gives an insight into the surgical technique and clinical follow-up. Treatment of osteochondral lesions or osteonecrosis by means of metal resurfacing implants is relatively new. During the past 3 years, promising clinical results were reported for the treatment of the femoral [24] and humeral head [18], as well as the first metatarsal [10] and patellar surface [7]. Two biomechanical cadaveric studies provided foundations for use of the talus implant in the ankle joint $[1,22]$.

The ideal indications for treatment with this implant are not yet known. The authors consider its use in patients with a large OCD on the medial talar dome who have clinically significant pain more than 1 year after primary surgical treatment. The following are regarded as contraindications: age $<18$ years, OCD size $>20 \mathrm{~mm}$, ankle osteoarthritis grade II or III, concomitant ankle pathology, advanced osteoporosis, infection, diabetes, or a known allergy to implant material.

The surgical approach is an important part of the implantation technique because the accuracy of implantation of this device strongly depends on the approach and quality of vision. For adequate exposure, the authors make an oblique osteotomy of the medial malleolus. The osteotomy is ideally directed toward the intersection between the tibial plafond and medial malleolus at an angle of $30^{\circ}$ relative to the long tibial axis [21]. Although there are other osteotomy options, including anterior tibial, step-cut, and inverted $\mathrm{V}$ or $\mathrm{U}$ osteotomies, the oblique osteotomy is our preferred technique because it is relatively simple, exposure of the talus is excellent, and congruent reduction is well possible $[12,17,21]$.

The surface of the prosthetic device should be placed slightly recessed relative to the surrounding surface of the talar cartilage because talar cartilage deforms during weight bearing while the implant does not. Wan et al. [25] measured a peak cartilage deformation of $34.5 \pm 7.3 \%$ under full body weight in persons with a medial talar dome cartilage thickness of $1.42 \pm 0.31 \mathrm{~mm}$. We therefore aim at an implantation level of $0.5 \mathrm{~mm}$ below the adjacent cartilage. This implantation level was found appropriate in a previous cadaveric study [22]. When the prosthetic device is correctly implanted, excessive contact pressures of the implant are avoided [22].

While there are various offset sizes, each articular component has a diameter of $15 \mathrm{~mm}$. This is based on the finding that primary arthroscopic treatment is generally successful for lesions up to $15 \mathrm{~mm}$, while this treatment is less successful for larger lesions [6]. The set diameter may be a disadvantage of the implant. In our patient, the largest defect diameter was $17 \mathrm{~mm}$. The part of the OCD that was not covered by the implant is expected to be filled by fibrocartilaginous tissue.

Alternative current treatment methods for this patient are osteochondral autograft transfer system (OATS), cancellous bone grafting, an allograft, ankle arthrodesis or prosthesis. OATS and cancellous bone grafting carry the risk of donor-site pain and are available in limited amounts $[19,2]$. Allografts can be used for massive defects but are not recommended for localized OCDs [20]. Ankle arthrodesis or prosthesis is definite solutions for a recurrent OCD but are rather not used in young patients. Recently reported other treatment options are matrix-induced autologous chondrocyte implantation (MACI) and engineered osteochondral grafts $[5,16]$. 
Fig. 3 Radiographs after 2 years follow-up. There are no implant-related complications or progressive degenerative changes of the ankle joint when compared to preoperatively

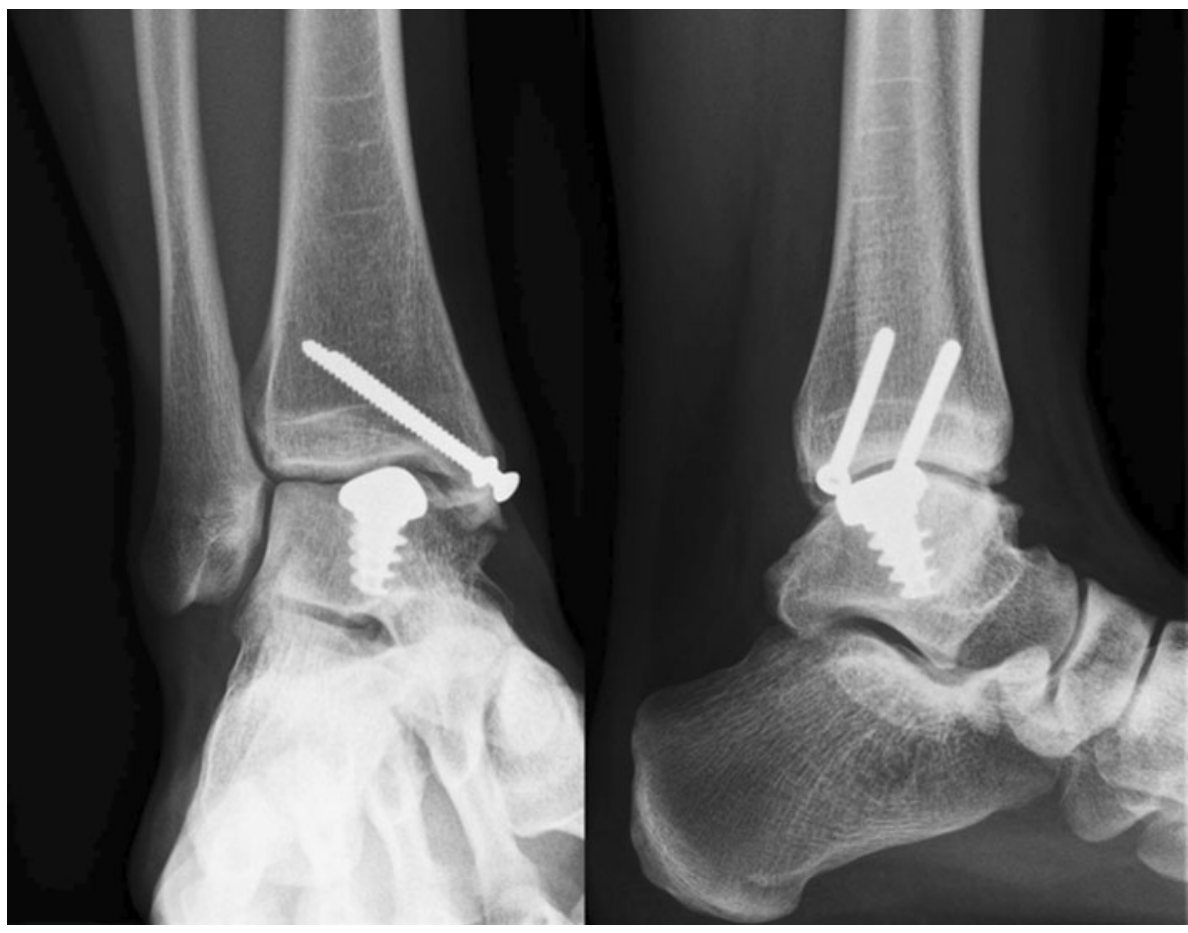

\section{Conclusion}

The metallic implantation technique appears to be a promising treatment for osteochondral defects of the medial talar dome after failed primary treatment. Although the clinical and radiological results of this prospective case report with 2 years follow-up are promising, more patients and longer follow-up are needed to draw any firm conclusions and determine whether the results continue with time.

Open Access This article is distributed under the terms of the Creative Commons Attribution Noncommercial License which permits any noncommercial use, distribution, and reproduction in any medium, provided the original author(s) and source are credited.

\section{References}

1. Anderson DD, Tochigi Y, Rudert MJ, Vaseenon T, Brown TD, Amendola A (2010) Effect of implantation accuracy on ankle contact mechanics with a metallic focal resurfacing implant. J Bone Joint Surg Am 92:1490-1500

2. Arrington ED, Smith WJ, Chambers HG, Bucknell AL, Davino NA (1996) Complications of iliac crest bone graft harvesting. Clin Orthop Relat Res 329:300-309

3. Banwart JC, Asher MA, Hassanein RS (1995) Iliac crest bone graft harvest donor site morbidity. A statistical evaluation. Spine 20:1055-1060

4. Baums MH, Heidrich G, Schultz W, Steckel H, Kahl E, Klinger HM (2006) Autologous chondrocyte transplantation for treating cartilage defects of the talus. J Bone Joint Surg Am 88:303-308

5. Candrian C, Barbero A, Bonacina E, Francioli S, Hirschmann MT, Milz S, Valderrabano V, Heberer M, Martin I, Jakob M
(2009) A novel implantation technique for engineered osteochondral grafts. Knee Surg Sports Traumatol Arthrosc 17:1377-1383

6. Chuckpaiwong B, Berkson EM, Theodore GH (2008) Microfracture for osteochondral lesions of the ankle: outcome analysis and outcome predictors of 105 cases. Arthroscopy 24:106-112

7. Davidson PA, Rivenburgh D (2008) Focal anatomic patellofemoral inlay resurfacing: theoretic basis, surgical technique, and case reports. Orthop Clin North Am 39:337-346

8. Gautier E, Kolker D, Jakob RP (2002) Treatment of cartilage defects of the talus by autologous osteochondral grafts. J Bone Joint Surg Br 84:237-244

9. Hangody L, Kish G, Modis L, Szerb I, Gaspar L, Dioszegi Z, Kendik Z (2001) Mosaicplasty for the treatment of osteochondritis dissecans of the talus: two to seven year results in 36 patients. Foot Ankle Int 22:552-558

10. Hasselman CT, Shields N (2008) Resurfacing of the first metatarsal head in the treatment of hallux rigidus. Tech Foot Ankle Surg 7:31-40

11. Kitaoka HB, Alexander IJ, Adelaar RS, Nunley JA, Myerson MS, Sanders M (1994) Clinical rating systems for the ankle-hindfoot, midfoot, hallux, and lesser toes. Foot Ankle Int 15:349-353

12. Navid DO, Myerson MS (2002) Approach alternatives for treatment of osteochondral lesions of the talus. Foot Ankle Clin 7:635-649

13. Paul J, Sagstetter A, Kriner M, Imhoff AB, Spang J, Hinterwimmer S (2009) Donor-site morbidity after osteochondral autologous transplantation for lesions of the talus. J Bone Joint Surg Am 91:1683-1688

14. Reddy S, Pedowitz DI, Parekh SG, Sennett BJ, Okereke E (2007) The morbidity associated with osteochondral harvest from asymptomatic knees for the treatment of osteochondral lesions of the talus. Am J Sports Med 35:80-85

15. Roos EM, Brandsson S, Karlsson J (2001) Validation of the foot and ankle outcome score for ankle ligament reconstruction. Foot Ankle Int 22:788-794 
16. Schneider TE, Karaikudi S (2009) Matrix-Induced Autologous Chondrocyte Implantation (MACI) grafting for osteochondral lesions of the talus. Foot Ankle Int 30:810-814

17. Seil R, Rupp S, Pape D, Dienst M, Kohn D (2001) Approach to open treatment of osteochondral lesions of the talus. Orthopade $30: 47-52$

18. Uribe JW, Botto-van BA (2009) Partial humeral head resurfacing for osteonecrosis. J Shoulder Elbow Surg 18:711-716

19. Valderrabano V, Leumann A, Rasch H, Egelhof T, Hintermann B, Pagenstert G (2009) Knee-to-ankle mosaicplasty for the treatment of osteochondral lesions of the ankle joint. Am J Sports Med 37(Suppl 1):105S-111S. doi:10.1177/0363546509 351481

20. van Bergen CJ, de Leeuw PA, van Dijk CN (2008) Treatment of osteochondral defects of the talus. Rev Chir Orthop Reparatrice Appar Mot 94:398-408

21. van Bergen CJ, Tuijthof GJ, Sierevelt IN, van Dijk CN (2010) Direction of the oblique medial malleolar osteotomy for exposure of the talus. Arch Orthop Trauma Surg. doi:10.1007/ s00402-010-1227-8

22. van Bergen CJ, Zengerink M, Blankevoort L, van Sterkenburg MN, van Oldenrijk J, van Dijk CN (2010) Novel metallic implantation technique for osteochondral defects of the medial talar dome. A cadaver study. Acta Orthop 81:495-502

23. van Dijk CN (2005) Osteochondral defect. In: Chan KM, Karlsson $\mathbf{J}$ (eds) ISAKOS-FIMS World consensus conference on ankle instability. Stockholm, Sweden, pp 68-69

24. Van Stralen RA, Haverkamp D, van Bergen CJ, Eijer H (2009) Partial resurfacing with varus osteotomy for an osteochondral defect of the femoral head. Hip Int 19:67-70

25. Wan L, de Asla RJ, Rubash HE, Li G (2008) In vivo cartilage contact deformation of human ankle joints under full body weight. J Orthop Res 26:1081-1089

26. Zengerink M, Struijs PA, Tol JL, van Dijk CN (2010) Treatment of osteochondral lesions of the talus: a systematic review. Knee Surg Sports Traumatol Arthrosc 18:238-246 